\title{
Tipos de mantenimiento aplicados en la industria petrolera venezolana de la Región Occidente
}

\author{
Types of maintenance applied in the Venezuelan oil industry of the \\ western region
}

\section{Renny Paredes \\ rennyparedesm@gmail.com \\ ORCID 0000-0002-3395-5669}

Universidad del Zulia, Núcleo Costa Oriental del Lago, Venezuela

Artículo recibido febrero 2020 | Arbitrado en marzo 2020 | Publicado en mayo 2020

\section{RESUMEN}

El estudio forma parte de una investigación de mayor alcance, cuyo objetivo general fue analizar la gestión de mantenimiento a taladros y gabarras en las divisiones de la industria petrolera venezolana en la región occidente. Este avance tuvo como propósito determinar los tipos de mantenimiento que se aplican en las mencionadas divisiones. La investigación fue descriptiva, con diseño no experimental, de campo y transeccional. Participaron cuatro divisiones, cuyas unidades informantes fueron los gerentes, líderes y supervisores. La técnica aplicada fue la encuesta y el instrumento un cuestionario, conformado por 12 ítems; validado por el juicio de expertos, con una confiabilidad muy alta de 0.89 según el método Alfa Cronbach. El análisis de los datos se realizó con estadística descriptiva, con base en la media aritmética. Se concluye que, estos tipos de mantenimiento: correctivo, preventivo, predictivo y proactivo arribaron a baja aplicación.

Palabras clave: correctivo; predictivo, preventivo, proactivo, tipos de mantenimiento

\begin{abstract}
The study is part of a larger investigation, whose general objective was to analyze the maintenance management of drills and barges in the divisions of the Venezuelan oil industry in the western region. The purpose of this advance was to determine the types of maintenance that are applied in the aforementioned divisions. The research was descriptive, with a non-experimental, field and transectional design. Four divisions participated, whose reporting units were managers, leaders and supervisors. The applied technique was the survey and the instrument a questionnaire, made up of 12 items; validated by expert judgment, with a very high reliability of 0.89 according to the Alpha Cronbach method. The data analysis was performed with descriptive statistics, based on the arithmetic mean. It is concluded that these types of maintenance: corrective, preventive, predictive and proactive arrived at low application.
\end{abstract}

Key words: corrective, predictive, preventive, proactive, types of maintenance 


\section{INTRODUCCIÓN}

En la actualidad, la actividad de mantenimiento ha sufrido un cambio en su concepción de trabajo, pasando a ser de una actividad reactiva, intensiva en tareas, a una concepción con enfoque eficiente $y$ proactivo, debido a que los paros imprevistos, son cada vez más costosos. Esto le proporciona a la actividad de mantenimiento una visión de negocio, ya que se convierte en un factor a tener en cuenta dentro de la estructura empresarial.

Por estas razones muchos empresarios y responsables de mantenimiento se preguntan si la dimensión, calidad y recursos de su gestión de mantenimiento, están en concordancia con los objetivos de su empresa. Es decir, desean conocer si su servicio de mantenimiento, será capaz ahora y en el futuro de lograr que la maquinaria e instalaciones trabajen con seguridad de funcionamiento, pero a un costo razonable y sostenible para la economía de la empresa.

Para poder asimilar estas inquietudes, es necesario conocer de manera precisa la situación en que se encuentra el estado actual de la gestión del mantenimiento, para tomar las medidas que sean necesarias; y la única manera de saberlo, es ejecutando un control y evaluación a los tipos de mantenimiento aplicados a taladros y gabarras en las divisiones de la industria petrolera venezolana en la región occidente.

En este marco referencial, para Duffuaa y otros (2010) los tipos de mantenimiento permiten a la organización conocer los requerimientos de cada máquina y equipo, para ello, es clave realizarlo dependiendo de las necesidades de la organización, logrando proporcionarle el servicio necesario para que la las actividades se realicen de acuerdo a lo establecido.

En este punto, las empresas en Venezuela no deberían quedar relegadas del resto del mundo. Las empresas y organizaciones, públicas y privadas, deben ocuparse en el desarrollo de nuevas formas para mejorar la optimización de los procesos productivos, es decir, mejoras al reducir costos operativos y aumentar los niveles de competitividad entre las empresas. Para ello, se fortalecen aplicando las mejoras en una gestión de mantenimiento en los procesos.

Al respecto, Martínez (2007) afirma que, el entorno económico e industrial revela la profundidad y amplitud del cambio en el ámbito productivo venezolano, tanto en maquinaria como en instalaciones, métodos de organización y trabajo, involucrando las actividades de mantenimiento, ya que algunas causas de fallas que se presentan son la expresión de falta de repuestos, personal no capacitado, distribución de un área de trabajo inadecuada, falta de información técnica de los equipos (manuales de operación y de mantenimiento), así como manual de partes de repuesto, servicios inadecuados (gas, energía, aire), reparaciones repetitivas al mismo equipo, falta de instrumentos de inspección, entre otros.

El reflejo de lo anterior, es lo que se percibe en la ejecución de sus labores de las divisiones de la industria petrolera venezolana en la región occidente, cuando se originan demoras e impuntualidades en el cumplimiento de los compromisos asumidos ante usuarios; congestionamiento en las entregas y recepciones de las solicitudes de mantenimiento a taladros y gabarras; reproceso de reparaciones que no 
se hicieron adecuadamente; variaciones constantes de los costos; uso de repuestos de menor calidad ante dificultades para importarlos.

En esta perspectiva, las divisiones de la industria petrolera venezolana en la región occidente, requieren de un plan estratégico que defina con claridad los tipos de mantenimiento necesarios en su gestión de mantenimiento acorde con las exigencias del mercado, garantizando la eficiente prestación del mantenimiento a taladros y gabarras.

\section{Gestión de mantenimiento}

La gestión de mantenimiento, según Duran (2010), debe ser un mecanismo valioso para asegurar que los principios de planificación total del ciclo de vida, gestión de riesgo, costo/beneficio, enfoque al cliente, sustentabilidad, entre otros, sean realmente implementados dentro del trabajo diario de desarrollo de proyectos de capital, operaciones, mantenimiento, u otros.

De modo tal, que los métodos relacionados con esta gestión, como señala este autor, deben asegurar que cada una de las piezas estén presentes y encajen adecuadamente considerando estos aspectos, es un juego de procesos, metodologías de confiabilidad integradas de forma ordenada y adoptada en tres fases muy detalladas para el cumplimiento de los objetivos empresariales (primera diagnóstico, segunda control y tercera, la optimización), los cuales están todos supeditados a herramientas, medidas de desempeños y entendimiento compartido.

En este marco de referencia, para Amendola (2006), la gestión de mantenimiento es una disciplina que se ha dado por muchos años, sobre todo cuando se expresa la frase de obtener el mayor retorno sobre la inversión. Hoy este término también es usado para describir la gerencia profesional de la infraestructura física, de datos e información, de gente, imagen pública, reputación y cualquier otro tipo de mantenimiento.

En este sentido, señala el autor citado, muchas compañías petroleras, de servicios $\mathrm{y}$ otras industrias han reconocido que a pesar de sus esfuerzos en reducir costos, nuevas tecnologías, iniciativas de calidad y productividad, la situación no es satisfactoria. Sigue siendo recurrente la ineficiencia, conflicto para cumplir, fallas de coordinación, y las oportunidades desaprovechadas.

De tal forma, Amendola (2006) señala que, la gestión de mantenimiento es la disciplina gerencial del enfoque de confiabilidad integral. De todas maneras, la gestión de mantenimiento provee técnicas para apoyar a quienes deben decidir tomando en cuenta innumerables factores técnicos, de seguridad, financieros, legales, laborales y restricciones sociales y ambientales; en otras palabras, la gestión de mantenimiento permite tomar decisiones sobre bases multicriterios.

Formalmente, la gestión de mantenimiento puede definirse, según Yañez y Gómez (2007), como un conjunto de disciplinas, metodologías y herramientas para optimizar el impacto sobre el ciclo de vida del negocio, de los costos, el desempeño y la exposición al riesgo, asociados con confiabilidad, disponibilidad, eficiencia, longevidad y cumplimiento de las regulaciones de seguridad y ambiente, de los activos físicos, en armonía con el activo humano.

De tal manera, afirman los autores citados, que si se establece una definición 
general de lo que puede entenderse como gestión de mantenimiento enfocada en activos físicos será como lo señalan los autores reseñados en el párrafo anterior: el juego de disciplinas, métodos, procedimientos $\mathrm{y}$ herramientas para optimizar el impacto total de costos, desempeño y exposición al riesgo en la vida del negocio asociados con confiabilidad, disponibilidad, mantenibilidad, eficiencia, longevidad y regulaciones de cumplimiento de seguridad.

Por su parte, Zambrano y Leal (2006) explican que, la gestión de mantenimiento debe ser un proceso sistémico donde a través de una serie de medidas organizativas se puedan planear las acciones de las actividades de mantenimiento (fases del proceso administrativo), esto con la finalidad de identificar los pasos a seguir (tipos de mantenimiento) y prever las posibles desviaciones que se puedan presentar durante el desarrollo de estas actividades de mantenimiento (elementos de la gestión).

En este sentido, la gestión de mantenimiento debe asegurar que todos los objetivos del negocio sean considerados y minimizar los conflictos entre ellos. Hay muchos intereses por satisfacer y algunos de ellos por naturaleza están en conflicto, por ejemplo máxima disponibilidad y mínimo costo. De modo que, esta gestión permite integrar las mejores prácticas en todos los aspectos de diseño, construcción, operación, mantenimiento y desincorporación de infraestructura física.

Ahora bien, en el Manual de mantenimiento de PDVSA (2005) se encamina como la estrategia operacional que, combinando procesos administrativos, disciplinas, métodos, herramientas informáticas, así como recursos técnicos, económicos y humanos, permite optimizar el impacto total en costos, el desempeño, y la exposición al riesgo durante el ciclo de vida del activo o del negocio.

Las situaciones descritas, por lo general están asociadas a aspectos específicos como integridad, confiabilidad, disponibilidad, mantenibilidad, eficiencia y eficacia, asegurando el logro de las metas operacionales, cumpliendo las regulaciones ambientales de salud ocupacional y de seguridad industrial. Además cuando se hace referencia a la gerencia o gestión significa mantener organizado y optimizado el mantenimiento de los activos y pasivos, los cuales pueden estar representados por edificios, maquinaria de planta, equipos y otros componentes permanentes requeridos por el usuario para producir y suministrar los productos, entre otros.

Por lo expuesto, el investigador asume que, la gestión de mantenimiento permite planificar, organizar, programar, ejecutar y controlar cada una de las actividades que se realizan a las máquinas y equipos, con la finalidad de ejecutar los procesos sobre la base de las necesidades planteadas en materia de mantenimiento, siendo efectivo en su ejecución para alcanzar los objetivos.

Por tanto, la gestión de mantenimiento, comprende el conjunto de actividades que se consideran en las organizaciones para lograr que las maquinarias, equipos e instalaciones funcionen de acuerdo a la planificación establecida, permitiendo la continuidad de los procesos.

\section{Tipos de mantenimiento}

Para Duffuaa y otros (2010) los tipos de mantenimiento han evolucionado con el tiempo, actualmente existen variados sistemas que pueden presentarse en las 
instalaciones de operación, los cuales exigen diferentes modos de mantenimiento para preservar la continuidad de los procesos productivos y sustentar la rentabilidad operacional, entre los cuales se encuentran: predictivo, preventivo, programado, correctivo y proactivo.

En este contexto, para Nava (2008), cuando se habla de tipos de mantenimiento, es más correcto hablar de tipos de tareas de mantenimiento, y en este sentido, existen diferentes clasificaciones de las tareas según distintos criterios. La clasificación más extendida se refiere a la naturaleza de las tareas, así, se han distinguido seis tipos de tareas de mantenimiento, que se diferencian entre sí por el carácter de las tareas que incluyen: correctivo, preventivo, conductivo, predictivo, cero horas, y modificativo.

Ahora bien, Jiménez y Milano (2006) son más específicos en este aspecto y afirman que, los tipos de mantenimiento se deben establecer con base en análisis económicos de criticidad y de riesgo de los equipos. Consideran, que para la buena ejecución y desempeño de las políticas de mantenimiento, se requiere una complementación de las diferentes categorías, buscando de esta forma una adecuada centralización de los distintos enfoques con sus características propias. De acuerdo a esta caracterización, se pueden realizar: mantenimiento correctivo, preventivo y predictivo.

En este orden de ideas, García (2009) afirma que la mayor parte de lo que se ha escrito hasta ahora sobre tipos de mantenimiento, hacen referencia, básicamente, a cuatro (4) tipos: correctivo, preventivo, predictivo y proactivo; a diferencia de los autores anteriores García agrega el tipo proactivo. Así, afirma García, en una organización estos tipos no son excluyentes; cuando se plantea la estrategia a seguir normalmente la respuesta es una combinación de los tipos de mantenimiento anteriores.

A su vez, considera este autor, que los tipos de mantenimiento señalados permiten coordinar, dirigir, organizar los recursos materiales, humanos y flujos de información destinados al correcto funcionamiento, reparación, así como prolongación de la vida de los equipos disponibles, para que, además, de lograr eficacia en las labores propias de mantenimiento se consiga una contención del gasto y la optimización de costos.

Con base en la exposición antes realizada, sobre los tipos de mantenimiento, para los fines de la presente investigación se consideró como base el criterio expuesto por García (2009) en lo concerniente a los tipos de mantenimiento, quien considera en conjunto cuatro (4) tipos: predictivo, preventivo, correctivo y proactivo. Esto a criterio del investigador, dado que forma parte del sector bajo estudio, divisiones de la industria petrolera venezolana en la región occidente, considerando que serían los de mayor pertinencia y aplicabilidad dentro del sector. Seguidamente serán objeto de análisis y se exponen detalladamente a continuación.

\section{Correctivo}

El tipo de mantenimiento correctivo, también denominado mantenimiento reactivo, tiene lugar luego que ocurre la falla o avería, es decir, solo actuará cuando se presenta un error en el sistema (Duffuaa y otros, 2010). En este caso si no se produce ninguna falla el mantenimiento será nulo, por lo que se tendrá que esperar hasta que se presente el desperfecto para recién 
tomar medidas de corrección de errores, lo cual trae consigo las siguientes consecuencias, según los autores citados:

- Paradas no previstas en el proceso productivo, disminuyendo las horas operativas. o Afecta las cadenas productivas, es decir, que los ciclos productivos posteriores se verán parados a la espera de la corrección de la etapa anterior.

- Presenta costos por reparación y repuestos no presupuestados, dado el caso que por falta de recursos no se podrán comprar repuestos en el momento deseado.

- El tiempo que estará el sistema fuera de operación no es predecible.

Por tanto, la acción de carácter puntual a raíz del uso, agotamiento de la vida útil u otros factores externos, de componentes, partes, piezas, materiales y, en general, de elementos que constituyen la infraestructura o planta, permitiendo su recuperación, restauración o renovación, no agrega valor al establecimiento.

En este marco de referencia, para Nava (2008) al tipo correctivo también se le conoce como mantenimiento reactivo, es aquel trabajo que involucra una cantidad determinada de tareas de reparación no programadas con el objetivo de restaurar la función de un activo una vez producido un paro imprevisto (parada forzada). Por su parte, Zambrano y Leal (2006) afirman que, las causas originarias de un paro imprevisto se deben a desperfectos no detectados durante las inspecciones predictivas, errores operacionales, ausencia de tareas de mantenimiento (reparaciones), sobre uso o utilización de los equipos fuera de las condiciones normales de operatividad del diseño, y a problemas de fabricación de partes o piezas de equipos.

Ahora bien, para García (2009), este tipo de mantenimiento puede presentar algunas ventajas como también desventajas. Entre las ventajas principales de esta estrategia señala: si el equipo está preparado, la intervención en el fallo es rápida y la reposición será con el mínimo tiempo; no se necesita una infraestructura excesiva, un grupo de operarios competentes será suficiente, por lo tanto el costo de mano de obra será mínimo, será más prioritaria la experiencia y la pericia de los operarios que la capacidad de análisis o de estudio del tipo de problema que se produzca; y es rentable en equipos que no intervienen de manera instantánea en la producción.

Mientras que las desventajas más predominantes serían: se producen paradas con daños imprevisibles en la producción que afectan a la planificación de manera incontrolada; se suele producir una baja calidad en las reparaciones debido a la rapidez en la intervención, y a la prioridad de reponer antes que reparar definitivamente, por lo que produce un hábito a trabajar defectuosamente, sensación de insatisfacción e impotencia, ya que este tipo de intervenciones a menudo generan otras al cabo del tiempo por mala reparación por lo tanto será muy difícil romper con esta inercia.

En síntesis, para el investigador, el mantenimiento correctivo es el conjunto de acciones necesarias para la realización de reparaciones o cambios de emergencia a nivel de los sistemas funcionales de la empresa o sistema productivo, al verse afectada la funcionalidad de un equipo o de sub-sistemas, ya sea por interrupción de sí misma o por la simple falla de equipos 
auxiliares de apoyo a la maquinaria principal.

\section{Preventivo}

El mantenimiento preventivo, se da antes de que ocurra la falla o avería y se efectúa en condiciones controladas, sin la existencia de algún error en el sistema; se realiza a razón de la experiencia, pericia del personal a cargo, los cuales son los encargados de determinar el momento justo para llevar a cabo el procedimiento; también se puede estipular el momento adecuado a través de manuales técnicos (Duffuaa y otros, 2010).

Estos autores lo definen como un proceso planificado, estructurado $\mathrm{y}$ controlado de tareas de mantenimiento a realizar dentro de las recurrencias establecidas, las mismas que generalmente son definidas por los fabricantes, y a falta de éstas se puede recurrir a las mejores prácticas del mercado de este tipo de servicios. Presenta las siguientes características, según Torres (2005):

- Se realiza en un momento en que no se está produciendo, por lo que se aprovechan las horas ociosas de la planta.

- Sigue un programa previamente elaborado, donde se detalla el procedimiento a seguir, actividades a realizar, a fin de tener las herramientas y repuestos necesarios.

- Cuenta con una fecha programada, además de un tiempo de inicio y de terminación preestablecido y aprobado por la directiva de la empresa.

- Está destinado a un área en particular, y a ciertos equipos específicamente. Aunque también se puede llevar un mantenimiento general de los componentes de la planta.

- Permite a la empresa contar con un historial de todos los equipos, además brinda la posibilidad de actualizar la información técnica de los equipos.

- Permite contar con un presupuesto aprobado por la directiva.

- Se aplica y se programa con el objeto de inspeccionar, reparar y cambiar parte de un equipo antes de ocurra la falla, eliminando o reduciendo los gastos.

Es importante señalar que según este concepto la lubricación, la limpieza, el ajuste de los equipos cuando trabajan, limpieza de la superficie, no son tareas del mantenimiento rutinario, por ello, es necesario realizarlo cuando corresponda mientras que se realiza el mantenimiento preventivo, esta teoría tiene que ser planteada y controlada.

Por su parte, Velásquez (2010) define el mantenimiento preventivo como una serie de tareas planeadas previamente que se llevan a cabo para contrarrestar las causas conocidas de fallas potenciales de las funciones para las que fue creado un activo. Puede planearse y programarse con base en el tiempo, el uso o la condición del tiempo. Mientras que para Parra (2012), el mantenimiento preventivo se define como el conjunto de acciones periódicas necesarias para conservar un equipo en buen estado. Independientemente del comportamiento o de la aparición de una falla se le dará a un equipo, y variará de acuerdo a su utilización y la necesidad de la producción del equipo.

Por lo antes planteado, a efectos del investigador, el mantenimiento preventivo queda definido como el conjunto de 
acciones o labores de mantenimiento que puedan ser previstas con suficiente anticipación, de tal forma que se planifiquen o se programen adecuadamente tanto en la forma y tiempo de ejecución. Es el que utiliza todos los medios disponibles, incluso estadísticos, para determinar la frecuencia de inspecciones, revisiones, sustitución de piezas claves, probabilidad de averías, vida útil, u otras.

\section{Predictivo}

El mantenimiento predictivo consiste en determinar en todo instante la condición técnica (mecánica y eléctrica) real de la máquina examinada, mientras ésta se encuentre en pleno funcionamiento, para ello se hace uso de un programa sistemático de mediciones de parámetros del equipo, según explican Duffuaa y otros (2010). El sustento tecnológico de este mantenimiento consiste en aplicaciones de algoritmos matemáticos agregados a las operaciones de diagnóstico, que juntos pueden brindar información referente a las condiciones del equipo. Tiene como objetivo disminuir las paradas por mantenimiento preventivos, $\mathrm{y}$ así minimizar los costos por mantenimiento y por no producción.

De acuerdo a Nava (2008), este tipo de mantenimiento se basa en predecir la falla antes de que ésta se produzca. Se trata de adelantarse al momento en que el equipo deja de trabajar en sus condiciones óptimas. Para lograrlo se utilizan herramientas y técnicas de monitores de parámetros físicos.

Involucra actividades que se desarrollan para detectar cualquier anomalía en equipos en funcionamiento, aplicando la tecnología para la interpretación de datos obtenidos a través de un diagnóstico, con instrumentos colocados en diferentes partes del equipo o bien sea por toma de muestras. Este tipo de mantenimiento no es dependiente de la característica de la falla, y es el más efectivo cuando el modo de falla es detectable por monitoreo de las condiciones de operación. Se lleva a cabo en forma calendario y no implica poner fuera de operación los equipos.

Para Mobley (2008) el mantenimiento predictivo es una técnica que utiliza la evaluación periódica de las condiciones de funcionamiento reales de los equipos de una planta, sus sistemas de producción y funciones de la gestión, para optimizar el funcionamiento total de la misma. La tecnología predictiva permite la evaluación precisa de todos los grupos funcionales, como el mantenimiento general dentro de la empresa. Utilizándose correctamente, el mantenimiento predictivo puede identificar no solo la mayoría, si no todos, los factores que limitan la eficacia y la eficiencia total de la planta (Mobley, 2008).

Por su parte, Zambrano y Leal (2006) afirman que, es el mantenimiento planificado y programado basándose en análisis técnicos y en la condición del equipo, antes de ocurrir una falla, sin detener el funcionamiento normal del equipo, para determinar la expectativa de vida de los componentes y reemplazarlos en tiempo óptimo. Se basa primordialmente en diseños electrónicos, son elementos adheridos a los sistemas.

En general, para el investigador, el mantenimiento predictivo consiste en estudiar la evolución temporal de ciertos parámetros y asociarlos a la evolución de las fallas, comúnmente, con alguna ayuda tecnológica, para así determinar en qué periodo de tiempo, esa falla va a tomar una relevancia importante, y así poder 
planificar todas las intervenciones con tiempo suficiente, para que no genere consecuencias negativas para los incides de productividad y eficiencia.

\section{Proactivo}

El mantenimiento proactivo comprende actividades que permiten aumentar la vida útil del equipo a través de un control de origen de la falla, utilizando las herramientas de un programa de monitoreo de condición, el cual se enfoca a las causas y no a los efectos de las mismas, a diferencia del mantenimiento predictivo (Torres, 2005).

También toma en cuenta las máquinas criticas de un sistema o instalación, análisis de modos de fallas, consecuencias, síntomas, efectos, y determina los objetivos de control para cada una de ellas, además de los tipos de análisis que se efectuarán y las medidas para volver a los valores establecidos. Su objetivo es extender la vida de la maquinaria, identificando la causa raíz que genera el desgaste, para mantener los parámetros de causa de falla dentro de límites aceptables.

Según Nava (2008), este tipo de mantenimiento es utilizado para mejorar el rendimiento de cualquier organización, considerando el estudio de probabilidad de falla, y disminuyendo el riesgo de aparición de la misma. Todo esto debe cumplirse para tratar de obtener costos totales mínimos de operación, operando en buenas condiciones durante un porcentaje de tiempo óptimo.

Ahora bien, García (2009) señala al tipo proactivo como una gran cantidad de técnicas para alargar la disponibilidad de las máquinas. Considera que la idea principal de un mantenimiento proactivo es el análisis de las causas fundamentales de las fallas, así estas causas se pueden remediar y los mecanismos de falla que se puedan eliminar gradualmente, mejoraran la operación de las máquinas. Visto así, el mantenimiento proactivo no se encarga de corregir los errores o defectos de las máquinas si no en lo que los causa. Lo que implicaría uno de los mantenimientos más baratos.

Por su parte, Alcalá (2008), considera que en este mantenimiento se utilizan técnicas especializadas para monitorear la condición de los equipos, basándose en el análisis de aceite para establecer el control de los parámetros de causa-falla. Asimismo, se establece una técnica de detección temprana, monitoreando el cambio en la tendencia de los parámetros considerados como causa-falla, para tomar acciones que permitan al equipo regresar a las condiciones establecidas y desempeñarse adecuadamente.

De acuerdo a esto, se puede señalar que este tipo de mantenimiento permite tener un control de máquinas y equipos, fortaleciendo los procesos mediante análisis de modos de fallas, criticidad, costos, extendiendo la vida útil de los equipos de la organización. Para el investigador, es clave considerar que la productividad aumentará en la medida en que las fallas de las máquinas disminuyan de forma sustentable en tiempo. Para lograrlo, resulta indispensable contar con la estrategia de mantenimiento más apropiada y personal capacitado tanto en el uso de las técnicas de análisis como en el diagnóstico de fallas implementadas, con conocimiento suficiente sobre las características de diseño y funcionamiento de las máquinas. 


\section{MÉTODO}

La investigación fue descriptiva, con diseño no experimental, de campo y transeccional. La población del estudio fueron las cuatro (4) divisiones de la industria petrolera venezolana en la región occidente, siendo éstas: División Costa Oriental, División Lago, División Sur del Lago-Trujillo y División Costa Occidental. Los sujetos informantes fueron gerentes generales de división, gerentes, líderes, supervisores mayores y supervisores, todos del área de mantenimiento a taladros y gabarras; para un total de 28 personas.

La técnica aplicada fue la encuesta y el instrumento un cuestionario, conformado por 12 ítems; validado por el juicio de expertos, con una confiabilidad muy alta de 0.89 según el método Alfa Cronbach. El análisis de los datos se realizó con estadística descriptiva, con base en la media aritmética. A tal fin se construyó un baremo, tal como se muestra en el cuadro 1.

Cuadro 1. Baremo para la interpretación de la media aritmética

\begin{tabular}{ccc}
\hline INTERVALO & CATEGORÍA & DESCRIPCIÓN \\
\hline $\begin{array}{c}\text { Muy alta aplicación } \\
3.41-4.21-50\end{array}$ & $\begin{array}{c}\text { Alta } \\
\text { aplicación }\end{array}$ & $\begin{array}{c}\text { Si la aplicación es muy alta o alta se considera una } \\
\text { fortaleza para la gestión de mantenimiento }\end{array}$ \\
$2.61-3.40$ & $\begin{array}{c}\text { Moderada } \\
\text { aplicación }\end{array}$ & $\begin{array}{c}\text { Si la aplicación es moderada se considera una leve } \\
\text { fortaleza, la gestión de mantenimiento requiere } \\
\text { mejoras }\end{array}$ \\
$1.81-2.60$ & $\begin{array}{c}\text { Baja } \\
\text { aplicación }\end{array}$ & $\begin{array}{c}\text { Si la aplicación es baja o muy baja se considera una } \\
\text { debilidad para la gestión de mantenimiento, por } \\
\text { tanto requiere mejoras. }\end{array}$ \\
\hline
\end{tabular}

\section{RESULTADOS}

En la tabla 1 se resume los resultados obtenidos para la dimensión tipos de mantenimiento, evidenciando una media de 2,56 implicando baja aplicación de los tipos de mantenimiento en la gestión de mantenimiento a taladros y gabarras que realizan las divisiones de la industria petrolera venezolana en la región occidente, delineándola como debilidades de la gestión, e indicando que la misma requiere mejoras. En resumen, todos los indicadores ostentan baja aplicación siendo debilidades de la gestión analizada, exceptuando el tipo de mantenimiento predictivo catalogado con moderada aplicación visto como una leve fortaleza; no obstante, en todos ellos se requieren mejoras de acuerdo al baremo diseñado. 
Tabla 1. Dimensión: Tipos de mantenimiento

\begin{tabular}{lll}
\hline INDICADORES & MEDIA & CATEGORÍA \\
\hline Correctivo & 2,46 & Baja aplicación/Debilidad \\
Preventivo & 2,25 & Baja aplicación/Debilidad \\
Predictivo & 3,15 & Moderada aplicación/Leve fortaleza \\
Proactivo & 2,38 & Baja aplicación/Debilidad \\
\hline DIMENSIóN & $\mathbf{2 , 5 6}$ & Baja aplicación/Debilidad \\
\hline
\end{tabular}

Estos resultados tienen baja reconocimiento de la importancia que debe congruencia con lo dicho por Jiménez y dársele a los tipos de mantenimiento. De tal Milano (2006), para quienes los tipos de forma, se está obviando que los tipos de mantenimiento se deben establecer con mantenimiento señalados permiten base en análisis económicos de criticidad y coordinar, dirigir, organizar los recursos riesgo de los equipos. Para la buena materiales, humanos y flujos de información ejecución y desempeño de las políticas de destinados al correcto funcionamiento, mantenimiento, se requiere una reparación, así como prolongación de la vida complementación de las diferentes de los equipos disponibles, para que, además categorías, buscando de esta forma una de lograr eficacia en las labores propias se adecuada centralización de los distintos consiga una contención del gasto y la enfoques con sus características propias. De optimización de costos. acuerdo a esta caracterización, se pueden realizar: mantenimiento correctivo, preventivo, predictivo y proactivo.

Lo mostrado permite al investigador afirmar que, en la gestión de mantenimiento a taladros y gabarras de las divisiones analizadas, no se posee un adecuado nivel de

En lo concerniente al detalle de cada uno de los indicadores utilizados, para medir la dimensión, de seguido se especifican los resultados obtenidos al respecto, mostrados en la tabla 2, la cual recoge el resultado de la dimensión y cada uno de sus indicadores.

Tabla 2. Indicadores: Tipos de mantenimiento

\begin{tabular}{|c|c|c|c|c|}
\hline INDICADOR & № & ÍTEMS & $\bar{X}$ & CATEGORÍA \\
\hline \multirow{4}{*}{ ن } & 1 & $\begin{array}{l}\text { Las acciones de mantenimiento se ejecutan } \\
\text { luego de ocurrida la falla del equipo. }\end{array}$ & 2.71 & $\begin{array}{c}\text { Moderada } \\
\text { aplicación/Leve } \\
\text { fortaleza }\end{array}$ \\
\hline & 2 & $\begin{array}{l}\text { Luego de una falla, se realiza un diagnóstico } \\
\text { para definir la causa raíz de la misma. }\end{array}$ & 2,04 & $\begin{array}{c}\text { Baja } \\
\text { aplicación/Debilidad }\end{array}$ \\
\hline & 3 & $\begin{array}{l}\text { Las fallas se controlan para evitar se afecte la } \\
\text { seguridad del ambiente }\end{array}$ & 2,64 & $\begin{array}{c}\text { Moderada } \\
\text { aplicación/Leve } \\
\text { fortaleza }\end{array}$ \\
\hline & & Promedio Indicador & 2,46 & $\begin{array}{c}\text { Baja } \\
\text { aplicación/Debilidad }\end{array}$ \\
\hline
\end{tabular}




\begin{tabular}{|c|c|c|c|c|}
\hline INDICADOR & № & ÍTEMS & $\bar{X}$ & CATEGORÍA \\
\hline \multirow{4}{*}{ 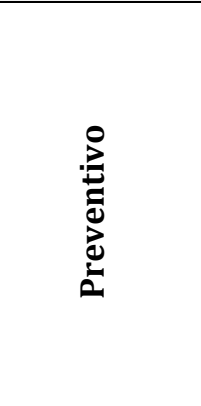 } & 4 & $\begin{array}{l}\text { Los mantenedores realizan inspecciones } \\
\text { periódicas a los equipos }\end{array}$ & 1,68 & $\begin{array}{c}\text { Muy baja } \\
\text { aplicación/Debilidad }\end{array}$ \\
\hline & 5 & $\begin{array}{l}\text { Realizan reemplazos de piezas a intervalos de } \\
\text { tiempo fijos en los equipos }\end{array}$ & 2,64 & $\begin{array}{l}\text { Moderada } \\
\text { aplicación/Leve } \\
\text { fortaleza }\end{array}$ \\
\hline & 6 & $\begin{array}{l}\text { Aplican técnicas para monitorear la condición } \\
\text { de funcionabilidad de los equipos. }\end{array}$ & 2,43 & $\begin{array}{c}\text { Baja } \\
\text { aplicación/Debilidad }\end{array}$ \\
\hline & & Promedio Indicador & 2,25 & $\begin{array}{c}\text { Baja } \\
\text { aplicación/Debilidad }\end{array}$ \\
\hline \multirow{4}{*}{ 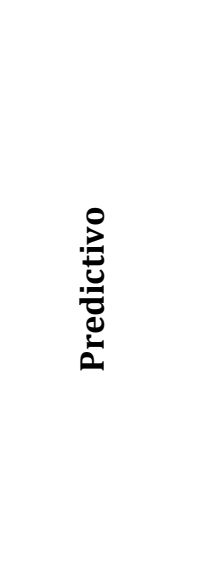 } & 7 & $\begin{array}{l}\text { Al ejecutar acciones de mantenimiento a los } \\
\text { equipos, se interrumpe el normal } \\
\text { funcionamiento en las unidades de medición }\end{array}$ & 3,11 & $\begin{array}{l}\text { Moderada } \\
\text { aplicación/Leve } \\
\text { fortaleza }\end{array}$ \\
\hline & 8 & $\begin{array}{l}\text { Las actividades de mantenimiento incluyen } \\
\text { análisis termo-gráfico de los componentes } \\
\text { asociados }\end{array}$ & 3,36 & $\begin{array}{l}\text { Moderada } \\
\text { aplicación/Leve } \\
\text { fortaleza }\end{array}$ \\
\hline & 9 & $\begin{array}{l}\text { Las actividades de mantenimiento ejecutadas } \\
\text { incluyen análisis de corrosión a los } \\
\text { componentes asociados }\end{array}$ & 3,00 & $\begin{array}{l}\text { Moderada } \\
\text { aplicación/Leve } \\
\text { fortaleza }\end{array}$ \\
\hline & & Promedio Indicador & 3,15 & $\begin{array}{l}\text { Moderada } \\
\text { aplicación/Leve } \\
\text { fortaleza }\end{array}$ \\
\hline \multirow{4}{*}{ 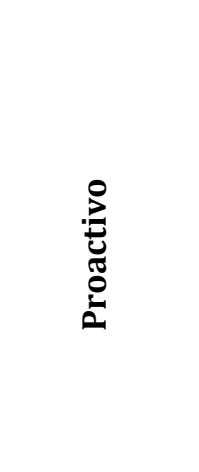 } & 10 & $\begin{array}{l}\text { Realizan actividades de mantenimiento que } \\
\text { permitan incrementar la vida útil de los } \\
\text { equipos mediante un control de la falla }\end{array}$ & 2,36 & $\begin{array}{l}\text { Baja } \\
\text { aplicación/Debilidad }\end{array}$ \\
\hline & 11 & $\begin{array}{l}\text { Establecen un control para cada una de las } \\
\text { máquinas que requiere mantenimiento }\end{array}$ & 2,21 & $\begin{array}{l}\text { Baja } \\
\text { aplicación/Debilidad }\end{array}$ \\
\hline & 12 & $\begin{array}{l}\text { Fortalecen los procesos mediante análisis de } \\
\text { modos de fallas. }\end{array}$ & 2,57 & $\begin{array}{c}\text { Baja } \\
\text { aplicación/Debilidad }\end{array}$ \\
\hline & & Promedio Indicador & 2,38 & $\begin{array}{c}\text { Baja } \\
\text { aplicación/Debilidad }\end{array}$ \\
\hline
\end{tabular}

De esta forma, se evidencia baja aplicación para los tipos de mantenimiento correctivo, preventivo y proactivo, ratificado con las medias alcanzadas de 2,$46 ; 2,25 ;$ y 2,38; respectivamente, indicando debilidades de la gestión; al tiempo que el mantenimiento predictivo se presenta con moderada aplicación, siendo una leve fortaleza para esta gestión; sin embargo, todos estos indicadores medidos están mostrando oportunidades de mejora, de acuerdo al baremo diseñado, por ello serán considerados en las acciones estratégicas a establecer en el siguiente objetivo específico.

Lo mostrado permite afirmar que, en la gestión de mantenimiento de las divisiones analizadas, no se han asumido los tipos de mantenimiento como la guía para obtener la calidad en sus procesos, productos $\mathrm{o}$ servicios, actuando al margen de lo descrito en la teoría base de esta investigación, como 
es el caso de García (2009) quien afirma que la mayor parte de lo que se ha escrito hasta ahora sobre tipos de mantenimiento, hacen referencia, básicamente, a cuatro (4) tipos: correctivo, preventivo, predictivo y proactivo; y en una organización estos tipos no son excluyentes; dado que cuando se plantea la estrategia a seguir normalmente la respuesta es una combinación de estos tipos.

De tal forma, los resultados no son coincidentes con Duffuaa y otros (2010) para quienes los tipos de mantenimiento permiten a la organización conocer los requerimientos de cada máquina y equipo, para ello, es clave realizarlo dependiendo de las necesidades de la organización, logrando proporcionarle el servicio necesario para que las actividades se realicen de acuerdo a lo establecido.

En este aspecto, a juicio del investigador, en las divisiones analizadas, se tiene poco claro el rol que deben jugar estos tipos de mantenimiento para el logro de una efectiva gestión, desconociendo tal vez que se han identificado estos cuatro (4) tipos como base del sistema de gestión de mantenimiento. Se debe tener presente que, estos tipos se desarrollan sobre la base de una planificación exhaustiva donde se considera el desempeño de los procesos operacionales, para lograr satisfacer las necesidades de todos los involucrados, mejorando constantemente los procesos que se emplean, estructura, personal, así como estrategias a implementar.

\section{CONCLUSIONES}

Se determinaron los tipos de mantenimiento que se aplican a taladros y gabarras en las divisiones de la industria petrolera venezolana en la región occidente, se concluye que: estos tipos de mantenimiento arribaron a baja aplicación, indicando que la gestión requiere mejoras. En detalle, todos los indicadores ostentaron baja aplicación, siendo considerados debilidades de esta gestión, exceptuando el tipo de mantenimiento predictivo catalogado con moderada aplicación visto como una leve fortaleza; no obstante, en todos los indicadores se requieren mejoras.

En este sentido, el investigador considera prudente:

- Velar porque se puedan realizar las acciones de mantenimiento al momento de ocurrir una falla del equipo.

- Establecer como política el control de las fallas para evitar se afecte la seguridad del ambiente.

- Implementar controles al ejecutar acciones de mantenimiento a los equipos, de manera que no se interrumpa el normal funcionamiento en las unidades de medición.

- Propiciar la realización de actividades de mantenimiento que permitan incrementar la vida útil de los equipos mediante un control de la falla.

\section{REFERENCIAS}

Alcalá, A. (2008). Introducción al mantenimiento. Segunda edición. Venezuela

Amendola, L. (2006). Gestión de proyectos de activos industriales. Editorial Universidad Politécnica de Valencia. Valencia. España

Duffuaa, S., Raouf, A. y Campbell, J. (2010). Sistemas de Mantenimiento. Planificación y Control. Editorial Limusa. México

Duran, J. (2010). Gestión de mantenimiento bajo estándares internacionales como PAS 55ASSET Management. The Woodhouse Parthership. En línea. Disponible en: 
http://www.mantenimientomundial.co $\mathrm{m} / \mathrm{sites} / \mathrm{mm} /$ notas/PAS55.pdf/

García, S. (2009). Organización y gestión integral de mantenimiento. Manual práctico para la implantación de sistemas de gestión avanzados de mantenimiento industrial. Ediciones Díaz de Santos, S.A. Madrid - España

Martínez, L. (2007). Organización y planificación de sistemas de mantenimiento. Centro de altos estudios gerenciales. Segunda Edición. Caracas. Venezuela

Milano (2005). La consultoría de empresas: Guía para la profesión. 2da. Edición. Editorial Limusa, S.A. México

Mobley, R. (2008). Maintenance Engineering Handbook. McGraw-Hill, Séptima Edición, New York, EEUU

Nava, J. (2008). Teoría del mantenimiento. Segunda Edición. Universidad de los Andes. Consejo de publicaciones. Mérida, Venezuela

Parra, C. (2012). Ingeniería de mantenimiento y fiabilidad aplicada a la gestión de activos. Ingeman. Primera Edición. Libro Electrónico

PDVSA (2005). Manual de Mantenimiento. Niveles de Mantenimiento PDVSA. Caracas, Venezuela

Torres, L. (2005). Mantenimiento, su implementación y su gestión. Universitas, Primera Edición, Libro Electrónico

Velásquez, J. (2010). Perspectivas modernas de la gestión del mantenimiento industrial. 4ta. Edición. Editorial Interamericana S.A. México

Yañez, M. y Gómez, H. (2007). Enfoque práctico para la estimación de confiabilidad y disponibilidad de equipos, con base en datos genéricos y opinión de expertos. Caracas, Venezuela

Zambrano, S. y Leal, S. (2006). Manual práctico de gestión de mantenimiento. Fondo Editorial UNET. San Cristóbal. Venezuela 